\title{
INFLUÊNCIA DAS REDES SOCIAIS VIRTUAIS NA SAÚDE DOS IDOSOS
}

\footnotetext{
Graciele Reinert Casadei ${ }^{1}$, Rose Mari Bennemann ${ }^{2}$, Tiago Franklin Rodrigues Lucena $^{3}$

${ }^{1}$ Mestranda em Promoção da Saúde pela Unicesumar, Maringá, Paraná, Brasil. gracielerei@hotmail.com

${ }^{2}$ Professora Dra. do Programa de Mestrado em Promoção da Saúde - Unicesumar.

Pesquisadora do Instituto Cesumar de Ciência, Tecnologia e Inovação - ICETI, Maringá, Paraná, Brasil.

${ }^{3}$ Professor Dr. do Programa de Mestrado em Promoção da Saúde Unicesumar.

Pesquisador do Instituto Cesumar de Ciência, Tecnologia e Inovação - ICETI, Maringá, Paraná, Brasil.

\section{Recebido em: 06/04/2019 - Aprovado em: 10/06/2019 - Publicado em: 30/06/2019} DOI: 10.18677/EnciBio_2019A152
}

\begin{abstract}
RESUMO
Atualmente, a população mundial está envelhecendo rapidamente. Em paralelo ao processo de envelhecimento, encontra-se o crescimento das novas tecnologias de comunicação e informação, como a Internet, que permite a interação entre as pessoas, inclusive idosos. No mesmo contexto, houve aumento significativo do número de idosos que estão acessando as redes sociais virtuais (RSV). Assim, este estudo teve como objetivo realizar uma revisão da literatura (janeiro de 2008 a abril 2018) sobre a influência das RSV na saúde dos idosos. Para realizar o levantamento, foram consultadas as bases de dados: PubMed, LILACS e SciELO. Após a triagem e avaliação, segundo os critérios de inclusão e exclusão, foram selecionados 34 artigos e cinco outros textos, como: leis, documentos, etc. Os artigos e textos destacam os benefícios que a inclusão digital e as RSV / Internet podem proporcionar aos idosos, dentre estes: comunicação, conhecimento, lazer, estímulo cognitivo e alteração da perspectiva de isolamento. Atuam diretamente na saúde, como subsídio para a promoção do bem-estar e da qualidade de vida, pois ajudam, por exemplo, a reduzir a depressão. No entanto, pesquisas com número significativo de idosos, bem delineadas, ou seja, que contenham risco mínimo de viés e que avaliem especificamente a intervenção RSV versus saúde dos idosos são necessárias. Além disso, os pesquisadores envolvidos nesta linha de pesquisa devem estar atentos às lacunas referentes a este assunto, como o desenvolvimento de estudos que verifiquem a longo prazo as consequências do uso das RSV / Internet na saúde dos idosos.
\end{abstract}

PALAVRAS-CHAVE: Internet, mídias sociais, Qualidade de vida.

THE INFLUENCE OF VIRTUAL SOCIAL MEDIA IN THE HEALTH OF ELDERLY: A REVIEW OF THE LITERATURE

\footnotetext{
ABSTRACT

Currently, world population is rapidly aging. Parallel to the population aging is the growth of the contemporary information and communication technologies, especially the Internet that allows interaction among people, including the elderly. In the same
} 
context, there was a significant increase in the number of elderly people accessing virtual social media (VSM). Thus, this study aimed to review the scientific literature (January 2008 to April 2018) on the influence of VSM in the elderly health. To perform this review, the databases PubMed, LILACS and SciELO were consulted. After screening and evaluation of the inclusion and exclusion criteria, were selected 34 articles and five other texts, such as: laws, dissertation, etc. The studies that were chosen for this review highlight the benefits that digital inclusion and VSM / Internet can offer the elderly, such as: communication, knowledge, leisure, cognitive stimulation and change in the perspective of isolation. They act directly in the health, well-being and quality of life, reducing depression. However, studies with a significant number of elderly, well-designed, containing a minimum risk of research bias and that assess specifically the intervention VSM versus elderly health are necessary. In addition, the researchers should be aware of gaps related to this subject, such as, the development of studies that verify the long-term consequences of the VSM / Internet use in the elderly health.

KEYWORDS: Social media, Internet, Quality of life.

\section{INTRODUÇÃO}

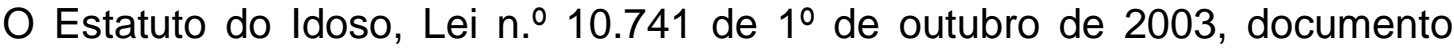
oficial da Federação Brasileira, define idosos como indivíduos com idade igual ou superior a 60 anos (BRASIL, 2003). Enquanto que a Organização Mundial da Saúde (OMS) define idosos como indivíduos com 65 anos ou mais, quando residentes em países desenvolvidos, ou com 60 anos ou mais, quando residentes em países subdesenvolvidos. Entretanto, independentemente da idade definida nos diferentes contextos citados, é relevante saber que a idade cronológica não é o único marcador para as mudanças que ocorrem com o envelhecimento, ou seja, existem alterações relacionadas ao estado de saúde (bem-estar físico, mental e social), participação e níveis de independência que diferem entre os idosos embora apresentem a mesma idade. Assim, um envelhecimento saudável é mais do que a ausência de doenças, envolve também a manutenção das habilidades funcionais e sociais dos idosos (OMS, 2015).

Atualmente, a população mundial está envelhecendo rapidamente, principalmente devido à queda da taxa de fecundidade associada ao aumento da expectativa de vida. Acompanhando a população mundial, a população brasileira também apresenta este fenômeno de envelhecimento. Entre os anos de 2012 e 2016, a população idosa no Brasil passou de 9,8\% para 14,3\%, chegando a 29,6 milhões (IBGE, 2016). De acordo com o Relatório Mundial sobre Envelhecimento e Saúde, no Brasil, o número de pessoas idosas aumentará de forma mais expressiva do que a média internacional. Calcula-se que o número de idosos no mundo duplicará até o ano de 2050, enquanto que no Brasil ela praticamente triplicará, transformando o país, consequentemente em uma "nação idosa" (OMS, 2015). Neste cenário, as pessoas vivendo mais e com saúde, principalmente nos grandes centros urbanos, tornam-se idosos que continuam ocupando espaço importante na sociedade, estudando, trabalhando, comprando, viajando e desenvolvendo projetos futuros (FELIZMINO; BARBOSA, 2018).

Em paralelo ao processo de envelhecimento da população, encontra-se o crescimento das inovações tecnológicas, como as novas tecnologias de comunicação e informação (NTICs), em especial a Internet, que permitem a interação entre as pessoas, atraindo públicos de diferentes idades, inclusive os idosos (AZEVEDO; CÔRTE, 2009). No mesmo contexto, houve aumento importante 
no número de idosos que estão utilizando as redes sociais virtuais (RSV) (MESSIAS, 2014). Em 2018, nos Estados Unidos, 37\% da população idosa (65 anos ou mais) utilizava RSV, destes $41 \%$ acessavam Facebook, 10\% Instagram, 9\% Linkedin e 8\% Twitter (PEW RESEARCH CENTER, 2018).

No Brasil, a pesquisa mais recente, realizada em 2016 pelo Serviço de Proteção ao Crédito (SPC Brasil) e pela Confederação Nacional de Dirigentes Lojistas (CNDL), mostrou que aproximadamente $50 \%$ dos idosos brasileiros (60 anos ou mais) usavam RSV e que $39 \%$ as utilizavam diariamente, sendo o Facebook a RSV mais acessada. A pesquisa também mostrou, que o emprego dos Smartphones é maior do que dos aparelhos tradicionais, ou seja, $61 \%$ navegavam usando celular, 53\% computadores tradicionais, 37\% notebook e 11\% Tablet (LIMA, 2016). Além disso, outra RSV que apresenta uso significativo entre os idosos é o WhatsApp, principalmente para manter contato com os familiares, para enviar fotos, compartilhar mensagens e para a participação de "grupos em comum" com os filhos, netos e parentes (DELLARMELIN et al., 2017; FERREIRA; TEIXEIRA, 2017).

Dentre os fatores que aguçam o interesse dos idosos em relação às RSV, está a vontade de pertencimento e inclusão na sociedade (FERREIRA; TEIXEIRA, 2017). Portanto, problematiza-se que certos aspectos psicossociais negativos, comuns aos idosos, como: solidão, isolamento social e alienação, podem ser minimizados com o apropriado uso das RSV e Internet, possibilitando a manutenção e / ou aprofundamento dos relacionamentos familiares / sociais, melhora dos níveis cognitivos, além de ser uma ferramenta útil na obtenção de informações sobre saúde, proporcionando, um "envelhecimento ativo" com melhor qualidade de vida e bem-estar (MIRANDA; FARIAS, 2009; PETERSEN et al., 2013; MARQUES et al., 2016; KRUG et al., 2017).

Tendo como base o contexto apresentado, este estudo teve como objetivo realizar uma revisão da literatura científica (janeiro de 2008 a abril 2018) sobre a influência das RSV na saúde dos idosos.

\section{LEVANTAMENTO DE DADOS}

Para realizar o levantamento dos estudos relacionados ao tema desta revisão, foram consultadas as seguintes bases de dados científicas: PubMed, LILACS e SciELO, utilizando para as buscas os descritores (em inglês no PubMed / em português no LILACS e SciELO): Elderly or older adults AND Internet / idosos e Internet; elderly or older adults AND virtual social media / idosos e redes sociais; social media AND older adults health AND Internet / redes sociais e idosos e saúde e Internet.

Como critérios de inclusão, foram adotados os seguintes itens: artigos ou trabalhos publicados no período de janeiro de 2008 a abril de 2018 (com exceção do estatuto do idoso), nos idiomas inglês ou português; artigos que tratavam de assuntos relacionados aos descritores selecionados e artigos ou trabalhos em que a versão completa estivesse com acesso livre (free access). Além disso, estudos referenciados nos artigos selecionados, que não estavam indexados nas bases de dados pesquisadas, mas que contemplavam os critérios deste estudo, também foram usados para o desenvolvimento desta revisão. Estudos que não se incluíam nos requisitos acima foram excluídos do trabalho. Assim, após a triagem foram incluídos, nesta revisão 34 artigos e cinco outros textos (uma lei, dois documentos: IBGE e OMS e duas pesquisas de opinião pública e análise de conteúdo midiático). 


\section{O IDOSO, A INCLUSÃO DIGITAL E A INTERNET}

No mundo moderno, as principais atividades econômicas, sociais, políticas e culturais podem ser facilmente mediadas pela Internet. Assim, as novas tecnologias fazem parte do cotidiano da população, tornando-a essencial para os novos modelos de vida que estão sendo impostos (AZEVEDO; CÔRTE, 2009). Fornecer ao idoso o acesso ao convívio social pelos diversos meios disponíveis, é um direito instituído pelo Estatuto do Idoso (Lei oㅜ 10.741 de 1ํ de outubro de 2003). O Artigo 21, Parágrafo Primeiro, garante que os cursos especiais para idosos devem incluir conteúdo concernente à comunicação, computação e demais avanços tecnológicos para sua integração com a vida contemporânea (BRASIL, 2003).

Os idosos estão cada vez mais ativos e consequentemente se inserindo gradativamente nos meios on-line e digitais, o que promove estímulo a cognição, possibilitando através desta conexão, melhorias na saúde e qualidade de vida destes indivíduos. $\mathrm{O}$ acesso a informação está mais fácil e rápido com o advento da tecnologia, concedendo a esta população ferramentas para exercitar a mente, com benefícios para memória e autoestima, integrando-se na vida do idoso, nas esferas social, afetiva e saúde mental (ALBAN et al., 2012; FONTANA; MARCHI, 2016). Diante disso, as tecnologias tornaram-se aliadas importantes no enfrentamento do medo e dos desafios, tornando o uso destes equipamentos um mecanismo de aprendizado intelectual (CRUZ et al., 2017).

Os idosos fazem parte de um mundo totalmente informatizado, no qual as informações estão disponíveis a todos, mas restrita aos que sabem acessá-las. O uso das novas tecnologias deixou de ser uma vantagem e passou a ser uma necessidade do dia a dia. Assim, a inclusão digital dos idosos visa suprir várias necessidades, visto que a informatização dos bancos, comércio e da vida moderna tem inibido os idosos de desenvolverem ações diárias, obrigando-os, na maioria das vezes, a precisarem da ajuda de terceiros para cuidar de seus interesses pessoais. Com o conhecimento, ainda que básico da informática e dispositivos tecnológicos, o idoso adquire maior independência, além de desenvoltura para manipular os equipamentos eletrônicos (PETERSEN et al., 2013).

Deve-se levar em consideração que a inclusão digital dos idosos compreende um processo diferente, quando comparados a crianças e jovens adultos, já que geralmente os idosos necessitam de tempo maior para manusear e assimilar as funcionalidades dos dispositivos tecnológicos. São várias as barreiras que dificultam a inclusão digital dos idosos, como a falta de contato com a tecnologia atual (muitos idosos consideram-se analfabetos digitais), associado a dificuldades do avanço da idade, como: declínios cognitivos, motores e físicos (TAVARES; SOUZA, 2012; SANTOS; ISHITANI; NOBRE, 2013; BRUNELLI et al., 2016; AZEVEDO, 2017). Em contrapartida, as novas gerações têm maior intimidade e atração pelos dispositivos tecnológicos, absorvem facilmente as mudanças, pois já convivem com a tecnologia desde pequenos, explorando os brinquedos eletrônicos e/ou brincando com celulares e tablets (SILVEIRA et al., 2017).

No Brasil, as barreiras a inclusão digital são inúmeras, como a baixa escolaridade, falta de projetos e programas sociais de inclusão digital, espaços de acesso aos computadores e internet oferecidos de forma gratuita, que podem ser mais graves, dependendo da região do país em que o idoso se encontra. A população idosa é a que sofre maiores prejuízos relacionados a essas barreiras, devido as alterações fisiológicas relacionadas ao processo de senescência, como a redução do tempo de reação, da memória de curto prazo, da atenção e concentração, da acuidade visual, da audição e da motricidade, tornando mais difícil 
o emprego da tecnologia. Além disso, constantemente surgem novas tecnologias e novas versões da mesma tecnologia, exigindo um aprendizado constante dos usuários. O estrangeirismo tanto do hardware como do software das tecnologias, é outro fator limitante que pode agravar o contexto da exclusão digital desses indivíduos. Portanto, a criação de alternativas de inclusão digital que pondere o ritmo, a linguagem e a história de vida dos idosos, são fundamentais para reduzir esses obstáculos (SALES et al., 2014).

Na revisão integrativa realizada por Souza e Sales (2016) sobre Tecnologias de Informação e Comunicação (TIC) utilizados por idosos na forma de smartphones e seus aplicativos, os autores verificaram que o objetivo dos diversos estudos foi atender às necessidades dos idosos por meio do desenvolvimento e avaliação de aplicativos e protótipos de interfaces para smartphones, afim de proporcionar benefícios a saúde, lazer, educação, entretenimento e também avaliar a usabilidade pelos idosos. Alguns deles, tanto nacionais quanto internacionais, constataram que se faz necessário para o correto manuseio do aplicativo por idosos, uma adequada capacidade cognitiva, sensorial e funcional.

A maioria das pesquisas são direcionadas para público jovem, no que se refere ao uso dos smartphones e raramente aos idosos. O número de idosos vem crescendo e juntamente com este o interesse pelo uso da tecnologia. Muitos idosos optam por não utilizarem essa ferramenta, tendo em vista os obstáculos encontrados frente ao manuseio da mesma. Essa realidade acaba promovendo a exclusão digital dos idosos, e mostra a necessidade de reflexão sobre as dificuldades encontradas na utilização das tecnologias pela população idosa (SOUZA; SALES, 2016; SILVEIRA et al., 2017).

Promover ações comunitárias e políticas públicas de inclusão digital aos idosos, de todas as classes sociais, é uma maneira de atender a legislação vigente e de oportunizar a eles o resgate da autonomia, autoestima, cidadania e do sentir-se útil (ORDONEZ et al., 2012; TAVARES; SOUZA, 2012). Petersen et al. (2013) relataram em seu estudo a experiência vivenciada com os idosos participantes de um projeto de inclusão social "A Melhor Idade na Era Digital". Este projeto, apesar de possuir como principal objetivo a orientação básica em informática para os idosos, tornou-se, segundo a observação dos autores, uma ferramenta de socialização e resgate da autoestima, mostrando que incluir socialmente não é apenas ensinar a utilização dos dispositivos tecnológicos, incluir é fornecer oportunidade ao indivíduo, para que ele desenvolva habilidades que facilitem a interação com a sociedade.

Seguindo uma linha de pesquisa semelhante, em um estudo mais recente, Brunelli et al., (2016) demonstraram que a inclusão digital dos idosos e o uso da Internet proporcionam melhora do contato social e familiar. Os idosos manifestaram também satisfação com o aprendizado e com as oportunidades que a inclusão digital proporcionou nas atividades diárias, no entretenimento, na preservação da saúde mental, na motivação para aprender o novo, na melhoria da memória e no rompimento de obstáculos impostos pela sociedade. Por conseguinte, a Internet pode ser considerada um dispositivo de inclusão social, que aumenta as possibilidades de interação social dos idosos (MESSIAS, 2014). Ao entender o seu funcionamento, os idosos adquirem conhecimentos que servirão de assunto e integração em futuros encontros com amigos e familiares, aproximando-os do mesmo universo dos filhos, netos e sobrinhos (JANTSCH et al., 2012).

Sendo assim, a inclusão digital e o correto uso da Internet fornecem aos idosos inúmeros benefícios como: comunicar-se através de RSV, e-mails e 
mensagens instantâneas, com parentes e amigos distantes com quem não tinham contato a muito tempo. No entanto, o contato virtual não exclui de modo algum o contato físico direto, na verdade, a comunicação digital permite aos idosos saberem mais sobre as pessoas que lhes são importantes e que muitas vezes não podem interagir com a frequência que gostariam (PETERSEN et al., 2013).

Além de reduzir distâncias, o uso da Internet favorece a vida social daqueles que acabam se isolando em suas residências, permitindo-lhes que de suas casas realizem compras e obtenham informações relacionadas a qualquer assunto (PETERSEN et al., 2013; SILVA et al., 2015), reduzindo o isolamento, melhorando a saúde e bem-estar (FERREIRA; ALVES, 2011; PEREIRA; NEVES, 2011; SILVEIRA et al., 2017).

As redes sociais, em geral, têm sido associadas de forma positiva à qualidade de vida e bem-estar. O convívio social dos idosos tende a ser menor com o avançar da idade e as redes sociais possibilitam aos idosos novos relacionamentos, contato com ambientes e situações diversas, promovendo manutenção ou melhora da qualidade de vida. $O$ envolvimento emocional é o fator de maior valorização relatada pelos idosos (GOUVEIA et al., 2016).

Medeiros et al. (2012) ao estudarem a relação entre inclusão digital, na forma de troca de mensagens pela Internet, e capacidade funcional de 1.656 idosos, entre 60 e 102 anos, residentes em Florianópolis - SC, demonstraram que os idosos que conseguiam enviar e receber mensagens pela Internet sem dificuldade apresentaram prevalência significativamente menor de dependência funcional moderada/grave, e que essa atividade, por sua vez, exerce um efeito benéfico sobre a capacidade cognitiva e o bem-estar social.

Em publicação mais recente, Krug et al. (2018) demonstraram em um estudo longitudinal com 1.197 idosos residentes em Florianópolis, que a prevalência do uso da Internet pelos idosos do projeto EpiFloripa foi elevada em relação à proporção de brasileiros na mesma faixa etária. Evidenciou também que a maioria dos idosos da pesquisa fazem uso da Internet diariamente ou quase todos os dias da semana, com o propósito de enviar e receber mensagens, pesquisar informações sobre saúde, pesquisar notícias e acessar RSV. Além disso, os idosos que tiveram maior chance de se manterem utilizando a Internet dentro dos quatro anos de estudo foram: do sexo masculino, com maior renda salarial, maior escolaridade e que não tinham cuidador.

Diante do exposto, o acesso à Internet e as RSV (das NTICs), é uma importante porta de acesso dos idosos a uma realidade que até o momento, era exclusiva aos jovens e adultos, proporcionando o desenvolvimento de uma geração de idosos mais ativos, conectados, com uma nova mentalidade e estilo de vida (PESSOA et al., 2008).

\section{REDES SOCIAIS VIRTUAIS E A SAÚDE DO IDOSO}

As Redes Sociais Virtuais (RSV) são espaços designados à socialização, utilizando para isso ferramentas de compartilhamento de informações, fotos, vídeos e comunicação entre seus usuários (WASSERMAN et al., 2012). Destacam-se como RSV mais utilizadas o Facebook, Instagram e Twitter. O uso destas RSV instiga os usuários a quererem saber quantas curtidas e/ou comentários suas postagens receberam, fazendo com que estes queiram conferir a todo momento o que está ocorrendo em seus perfis (PICON et al., 2015). Assim, as RSV podem ser consideradas um espaço de ressocialização para os idosos que as utilizam, pois dispõem de diversas ferramentas para interações e comunicação, fornecendo aos 
idosos a oportunidade de remodelar sua representação social (CARVALHO et al., 2014; DELLARMELIN; FROEMMING, 2015).

O crescimento do uso das RSV pelos idosos provoca investigações sobre o uso das mesmas. Torna-se, portanto, importante que se compreenda as RSV como um espaço capaz de proporcionar aos idosos interagir e gozar das possibilidades que sejam a eles oferecidas (MESSIAS, 2014). Com grande representatividade, 0 Facebook é uma das RSV mais utilizadas por idosos em todo o mundo, como um espaço de encontro, partilha, interação e discussão de ideias e temas de interesse em comum ( JANTSCH et al., 2012; WASSERMAN et al., 2012; DELLARMELIN et al., 2017; PEW RESEARCH CENTER, 2018). Segundo Wasserman et al. (2012), o maior uso do Facebook por idosos ocorre por ser intuitivo e se tratar de um espaço de fácil utilização, ou seja, é um espaço que adota recursos com conteúdo na língua portuguesa (como jogos), fácil de postar e se comunicar, além do uso do popular 'Curtir', uma maneira prática de manifestação.

As ferramentas mais utilizadas no Facebook pelos idosos são, as mensagens instantâneas (Messenger), seguido do compartilhamento de fotos. Além disso, a preocupação dos idosos com a privacidade nas RSV é significativa, e este receio ocorre devido a exposição da vida pessoal. A facilidade em fazer "amizades" nos ambientes virtuais intimida os idosos, uma vez que esta parte da população está acostumada a construir amizades presenciais baseadas em conversas e confiança, diferentemente das desenvolvidas nas RSV (WASSERMAN et al., 2012).

Estes resultados corroboram com dados mais recentes obtidos por Dellarmelin et al. (2017). Estes autores apontam em sua pesquisa que todos os 191 idosos entrevistados, do município de Passo Fundo -RS, têm acesso à internet e que as RSV mais acessadas são Facebook, WhatsApp, Twitter, Instagram e SnapChat, respectivamente. Além disso, a maioria dos idosos entrevistados utilizam as RSV por até quatro horas por dia, o principal motivo para a criação de conta em alguma RSV é poder conversar com outras pessoas, principalmente para manter contato com familiares ou amigos distantes. Ainda segundo os autores, os idosos estudados não consideram as redes sociais seguras, sendo este um dos motivos para explorar principalmente ferramentas que propiciem diálogos.

E importante destacar que situação de aposentadoria, perda do cônjuge ou amigos (encolhimento do tamanho da rede social), morar sozinho ou em instituições e mudanças físicas (deficiências cognitivas e motoras), levam os idosos a apresentarem uma perda da qualidade de vida em virtude da diminuição das atividades intelectuais, sociais e físicas, o que favorece muitas vezes quadros de depressão que agravam significativamente doenças pré-existentes e mortalidade (BATISTA et al., 2015; CHEN; SCHULTZ, 2016; SILVEIRA et al., 2017). Em seu estudo, Cotten et al. (2014) demonstraram uma contribuição positiva do uso da Internet / RSV para o bem-estar mental de idosos aposentados nos Estados Unidos. Segundo os autores, o uso da Internet / RSV reduziu a probabilidade de um estado deprimido em cerca de $33 \%$ dos idosos, principalmente daqueles que vivem sozinhos.

Por este motivo, convívio social ativo, seja virtual ou presencial, favorece a qualidade de vida dos idosos (SILVEIRA et al., 2017). Concernente aos ganhos em nível do cognitivo - capacidades sensoriais, diminuição e desgaste da memória e dificuldade em fixar informações - os idosos têm tornado o computador e a Internet um grande aliado para estimular a memória, construir e aprimorar os conhecimentos, para a criatividade e inteligência (WASSERMAN et al., 2012). Estudo realizado por Krug et al. (2017) reforçam os dados expostos acima, estes autores demonstraram 
em um estudo prospectivo de intervenção controlada, realizado com 293 idosos, que a intervenção mediados por computadores e Internet (usar o mouse, ferramentas de desenho, jogos, e-mail, RSV ...) estão associados a melhora do status cognitivo.

A interação com as RSV serve de estímulo para aquisição de novos hábitos, trazendo diversos benefícios para a estimulação dos idosos, como a retirada do ócio, tornando-se lazer e distração, fazendo com que se sintam parte integrante do novo estilo de sociedade, bem como saiam do sedentarismo (DELLARMELIN; FROEMMING, 2015). Skura et al. (2013) ao estudarem um grupo de idosos, demonstraram que a inserção no ambiente virtual influencia na melhoria da qualidade de vida, sob o aspecto de saúde física, principalmente porque muitos idosos, procuram informações sobre saúde dentre as notícias publicadas. Este dado pode ser um indicativo de que é viável o uso das RSV e da Internet como ferramenta para a promoção da saúde em forma de notícia ou outros tipos de postagens informativas.

Com o advento da internet, os idosos e a população em geral, passaram a adquirir mais autonomia e independência sobre os cuidados necessários com suas doenças e tratamentos, visto que a informação auxilia neste processo de assimilação das informações fornecidas pelos médicos e por outros profissionais de saúde, consideradas escassas na maioria das vezes, por grande parte da população (MANSO et al., 2017; SANTOS et al., 2017; AMORIM et al., 2018). Além disso, o uso da internet, proporciona a troca de experiências entre os idosos relacionadas as informações adquiridas, como também a averiguação da veracidade das mesmas. Atualmente na internet, encontram-se grupos de apoio de diversas comunidades de doentes, que permite a divulgação de informações científicas e a troca de experiências entre os idosos, relacionada aos tratamentos realizados e evolução da doença. Esses grupos aumentam o bem-estar emocional e têm sido reconhecidos como um veículo de compartilhamento de sensações sobre doença e saúde, visto que os estudos demonstram essa correlação positiva entre os participantes dos grupos, possibilitando o empoderamento pessoal (MANSO et al., 2017).

Um estudo realizado no período de março de 2017 a setembro de 2017 selecionou 141 publicações de um site de relacionamento para idosos, o blog "Portal da Terceira Idade", com a finalidade de compreender os sentimentos de solidão e a busca por companhia. Dentre as publicações encontradas, 107 mostraram interesse em um relacionamento amoroso, 30 buscaram apenas amizade e quatro eram divulgações relacionadas a atividades em grupo, com o objetivo de promover a inclusão, através de excursões e outras atividades. Os idosos expressaram sentimentos de solidão na maioria dos comentários, sendo que grande parte eram viúvos ou separados, com filhos distantes morando em outras cidades. Observou-se que os sentimentos de solidão são frequentes nos idosos, visto que esse sentimento gera sofrimento, promove consequentemente a redução da qualidade de vida destes indivíduos. O estudo verificou que a tecnologia pode favorecer a busca por relacionamentos entre idosos, diminuindo o sentimento de solidão e abandono, servindo como ferramenta facilitadora na busca por companhia, permitindo a promoção de um envelhecimento saudável (LUCIANO, 2018).

Chen e Schultz (2016) em uma revisão sistemática da literatura científica demonstraram que as novas tecnologias de informação e comunicação (NTICs), como por exemplo, a Internet, podem ser uma ferramenta para combater o isolamento social entre os idosos, através de quatro mecanismos principais: conexão com o mundo exterior, apoio social, envolvimento em atividades de interesse e aumento da autoconfiança. Apesar disso, os resultados desta revisão 
sugerem que o uso das NTICs não garante a qualidade da comunicação. Quando a comunicação não é recíproca, por exemplo, o seu uso pode aumentar o isolamento social entre os idosos.

A presença de contradições entre os estudos, levam a resultados inconclusivos das NTICs no impacto da redução da solidão, sugerindo que estes recursos não são adequados para todos os idosos. Estudos mais bem delineados que apresentem risco mínimo de viés de pesquisa, são necessários para tirar conclusões assertivas sobre a real eficácia das NTICs para idosos na redução do isolamento social, tendo em vista que o isolamento social deve ser percebido como um conceito multidimensional que envolve o número de contatos, o sentimento de pertencimento, a presença de relacionamentos satisfatórios, o grau de envolvimento com os outros e a qualidade dos membros da rede (CHEN; SCHULTZ, 2016).

Além disso, até hoje, são poucos os estudos sobre o potencial negativo e consequências do uso das RSV e Internet por idosos. As principais consequências potencialmente negativas, que podem também envolver qualquer faixa etária, são: ameaças à saúde e ao bem-estar do idoso decorrentes da adoção de informações prejudiciais, comportamentos prejudiciais e uso indevido de informações pessoais dos idosos compartilhadas por criminosos (LEIST, 2013). No tocante à dependência, as RSV são o desdobramento da dependência da Internet. Felizmino e Barbosa (2018) através de uma revisão bibliográfica, constataram a atual insuficiência de estudos evidenciando (ou não) o desencadeamento deste transtorno nos idosos. Assim, ignorar o fenômeno de que os idosos, podem, assim como os demais grupos etários, desencadearem o transtorno de dependência, é desconsiderar a suscetibilidade para este adoecimento. Haja vista, que alguns fatores relevantes enfrentados por alguns idosos devem ser considerados nesse contexto como: ociosidade e sedentarismo pós-aposentadoria, solidão e/ou desconhecimento dos riscos do mundo virtual.

A geração atual que caminha para o envelhecimento, ou seja, a próxima geração de idosos, estará mais familiarizada com os recursos tecnológicos aos quais os idosos atualmente estão se adaptando (PESSOA et al., 2008). Assim, as pesquisas futuras terão que mudar o foco, de primeiros encontros nas RSV e Internet para mudanças e consequências a longo prazo do uso frequente das RSV e Internet por idosos (LEIST, 2013).

\section{CONSIDERAÇÕES FINAIS}

As redes sociais virtuais são uma realidade e temática relativamente recente que ainda necessita de mais estudos. Pode-se observar que embora a maioria dos estudos relatem que as redes sociais exercem efeito positivo sobre a saúde do idoso, melhorando a qualidade de vida, ainda são poucas as pesquisas sobre as consequências do uso inadequado das RSV relacionadas aos aspectos físico, mental e social. Além disso, são necessários estudos que verifiquem a longo prazo as consequências, positivas e negativas, do uso da Internet e das Redes Sociais Virtuais na vida dos idosos.

\section{REFERÊNCIAS}

ALBAN, A.; MARCHI, A. C. B. de; SCORTEGAGNA, S. A.; LEGUISAMO, C. P. Ampliando a usabilidade de interfaces web para idosos em dispositivos móveis: uma proposta utilizando design responsivo. Revista Novas Tecnologias na Educação, v. $10, \quad$ n. $3, \quad$ p. 1-10, 2012. Disponível em: <https://seer.ufrgs.br/renote/article/view/36404/23511>. 
doi: https://doi.org/10.22456/1679-1916.36404.

AMORIM, D. N. P.; SAMPAIO, L.V.P.; CARVALHO, G.A.; VILAÇA, K.H.C. Aplicativos móveis para a saúde e o cuidado de idosos. Revista Eletrônica de Comunicação, Informação \& Inovação em Saúde, Rio de Janeiro, v. 12, n. 1, p. 58-71, jan./mar. 2018.

Disponível

em:

<https://www.reciis.icict.fiocruz.br/index.php/reciis/article/view/1365>.

doi: http://dx.doi.org/10.29397/reciis.v12i1.1365.

AZEVEDO, C. D.; CÔRTE, B. Breve reflexão sobre a Internet e a longevidade: novos espaços de socialização preparam o silêncio da saúde. In: SESCSP. A terceira idade: estudos sobre envelhecimento, São Paulo: Sesc-Geti, v. 20, n. 45, p. 7-37, 2009. Disponível em:

https://www.sescsp.org.br/files/edicao_revista/6850d706-40ce-4790-8931c4f13f8f8af0.pdf.

AZEVEDO, C. TIC e sociedades cada vez mais envelhecidas: uma contextualização de estudos no Brasil, em Portugal e em outros países. Verso e Reverso, São Leopoldo, v. 31, n. 76, p. 14-25, jan./abr. 2017. Disponível em: <http://revistas.unisinos.br/index.php/versoereverso/article/view/ver.2016.31.76.02>. doi: 10.4013/ver.2016.31.76.02

BATISTA, M. P. P.; SOUZA, F.G.; SCHWARTZ, G.; EXNER, C.; ALMEIDA, M.H. M.; Utilização no cotidiano de tecnologias da informação e comunicação por idosos participantes da Universidade Aberta da Terceira Idade da Universidade de São Paulo. Revista Kairós Gerontologia, São Paulo, v. 18, n. 4, p. 405-426, 2015. Disponível em: https://revistas.pucsp.br/index.php/kairos/article/view/30257.

BRASIL. Lei n. 10.741, de $1^{\circ}$ de outubro de 2003. Dispõe sobre o Estatuto do Idoso e dá outras providências. Estatuto do Idoso; Lei do Idoso. Diário Oficial da União, Poder Executivo, Brasília, 2003. Disponível em:

https://www2.camara.leg.br/legin/fed/lei/2003/lei-10741-1-outubro-2003-497511publicacaooriginal-1-pl.html.

BRUNELLI, A. V.; CHICON, P. M. M.; EICH, S. C.; KUSCHEL, C. F.; PREVEDELLO, J. D. et al. Uma parceria na inclusão digital. Revista de Extensão da Universidade de Cruz Alta, v. 8, n. 1, p. 321-331, 2016. Disponível em: file://C:/Users/ADM/Downloads/4074-13556-2-PB.pdf.

CARVALHO, G. M.; TARALLO, R. dos S.; BATISTONI, S. S. T.; CACHIONI, M. Redes sociais e geratividade: A experiência do programa idosos on-line. Estudos interdisciplinares sobre o envelhecimento, v. 19, n. 3, p. 793-812, 2014. Disponível em: https://seer.ufrgs.br/RevEnvelhecer/article/view/40759/33286.

CHEN, Y- R. R.; SCHULZ, P. J. The effect of information communication technology interventions on reducing social isolation in the elderly: A systematic review. Journal of Medical Internet Research, v. 1 8, n. 1, p. 1-31, 2016. Disponível em: <https://www.ncbi.nlm.nih.gov/pmc/articles/PMC4751336/>.

doi: 10.2196/jmir.4596 
COTTEN, S. R.; FORD, G.; FORD, S.; HALE, T. M. Internet use and depression among retired older adults in the United States: a longitudinal analysis. The Journals of Gerontology: Series B, v. 69, n. 5, p. 763-771, 2014. Disponível em: <https://academic.oup.com/psychsocgerontology/article/69/5/763/2940062>. doi: https://doi.org/10.1093/geronb/gbu018.

CRUZ, A.; BEULCL, L.; TEIXEIRA, L.; SOUZA, L.; SANTANA, V. et al. Consumo das redes sociais digitais pela terceira idade. In: Anais do 40 Congresso Brasileiro de Ciências da Comunicação, Curitiba-PR, set. 2017, p. 1-14. Disponível em: http://portalintercom.org.br/anais/nacional2017/resumos/R12-2629-1.pdf.

DELLARMELIN, M. L.; FROEMMING, L. M. S. Vovôs conectados: análise da utilização das redes sociais pelos idosos. XV Mostra de Iniciação Científica, Pós Graduação, Pesquisa e Extensão da UCS, Caxias do Sul, RS, p. 1-10, 2015. Disponível

em: <http://www.ucs.br/etc/conferencias/index.php/mostraucsppga/xvmostrappga/paper/v iewFile/4195/1375>.

DELLARMELIN, M. L.; BALBINOT, V. A.; FROEMMING, L. M. S. Análise do comportamento e utilização das redes sociais pelos idosos. Revista Sociais e Humanas, v. 30 , n. 1, p. 174-184, 2017. Disponível em: $<$ https://periodicos.ufsm.br/sociaisehumanas/article/view/24669/pdf>.

doi: $10.5902 / 2317175824669$.

FELIZMINO, T. de O.; BARBOSA, R. B. Idosos e dependência de internet: uma revisão bibliográfica. Revista Psicologia, Diversidade e Saúde, v. 7, n. 1, p.139146, 2018.2 Disponível em: <https://www5.bahiana.edu.br/index.php/psicologia/article/view/1669/1682>. doi: 10.17267/2317-3394rpds.v7i1.1669.

FERREIRA, M. A. S.; ALVES, V. P. Representação social do idoso do Distrito Federal e sua inserção social no mundo contemporâneo a partir da Internet. Revista Brasileira Geriatria e Gerontologia, v. 14, n. 4, p. 699-712, 2011. Disponível em: $<$ http://www.scielo.br/pdf/rbgg/v14n4/a09v14n4.pdf>.

doi: http://dx.doi.org/10.1590/S1809-98232011000400009.

FERREIRA, M. C.; TEIXEIRA, K. M. D. O uso de redes sociais virtuais pelos idosos. Estudos Interdisciplinares sobre o Envelhecimento, Porto Alegre, v. 22, n. 3, p. 153-167, $2017 . \quad$ Disponível em: https://seer.ufrgs.br/RevEnvelhecer/article/view/74595/49695.

FONTANA, E.; MARCHI, A. C. B. de. Aplicativos para treino cognitivo: uma revisão sistemática. Revista Novas Tecnologias na Educação, Porto Alegre, v. 14, n. 2, dez. 2016. Disponível em: <https://seer.ufrgs.br/renote/article/view/70651>. doi: https://doi.org/10.22456/1679-1916.70651.

GOUVEIA, O. M. R.; MATOS, A. D.; SCHOUTEN, M. J. Redes sociais e qualidade de vida dos idosos: uma revisão e análise crítica da literatura. Revista Brasileira de Geriatria e Gerontologia, v. 19, n. 6, p. 1030-1040, 2016. Disponível em: <http://www.scielo.br/pdf/rbgg/v19n6/pt_1809-9823-rbgg-19-06-01030.pdf>. 
doi: http://dx.doi.org/10.1590/1981-22562016019.160017.

INSTITUTO BRASILEIRO DE GEOGRAFIA E ESTATÍSTICA (IBGE). Síntese de indicadores sociais: uma análise das condições de vida da população brasileira. Rio de Janeiro: IBGE, 2016. 146 p. Disponível em: $<$ https://biblioteca.ibge.gov.br/visualizacao/livros/liv98965.pdf>.

JANTSCH, A.; MACHADO, L. R.; BEHAR, P. A.; LIMA, J. V. de. As redes sociais e a qualidade de vida: os idosos na era digital. Revista lberoamericana de Tecnologías del Aprendizaje, v. 7, n. 4, p. 173-179, 2012. Disponível em: http://rita.det.uvigo.es/201211/uploads/IEEE-RITA.2012.V7.N4.A2.pdf.

KRUG, R. de R.; SILVA, A. Q. A. D.; SCHNEIDER, I. J. C.; RAMOS, L. R.; D'ORSI, E. et al. Cognitive cooperation groups mediated by computers and internet present significant improvement of cognitive status in older adults with memory complaints: a controlled prospective study. Arquivos de Neuropsiquiatria, v.75, n.4, p. 228- 233, 2017. Disponível em: <https://www.ncbi.nlm.nih.gov/pubmed/28489142>. doi: 10.1590/0004-282X20170021.

KRUG, R. de R.; XAVIER, A. J.; D'ORSI, E. Fatores associados à manutenção do uso da internet, estudo longitudinal EpiFloripa Idoso. Revista de Saúde Pública, v. 52, n. 37, p. 1-12, 2018. Disponível em: <http://www.scielo.br/scielo.php?pid=S0034$89102018000100230 \&$ script=sci_arttext\&tlng=pt $>$.

doi: http://dx.doi.org/10.11606/s1518-8787.2018052000216.

LEIST, A. K. Social Media Use of Older Adults: A Mini-Review. Gerontology, v. 59, n. $4, \quad$ p. 378-384, 2013. Disponível em: <https://www.karger.com/Article/FullText/346818>. doi: 10.1159/000346818.

LIMA, M. Facebook é a rede social mais usada por idosos. Veja, São Paulo, 18 out. 2016. Disponível em: <https://veja.abril.com.br/blog/radar/facebook-e-a-rede-social-maisusada-por-idosos/>. Acesso em: 1 jan. 2019.

LUCIANO, A. C. de L. Redes virtuais: uma alternativa à solidão? Relacionamentos na velhice. Revista Portal de Divulgação, n. 56, p. 5-10, 2018. Disponível em: http://www.portaldoenvelhecimento.com/revistanova/index.php/revistaportal/article/view/709/772.

MANSO, M. E. G.; ROTH, M. C.; LOPES, R. G. da C. Idosos vivenciando o diabetes nas redes sociais. Revista Portal de Divulgação, n. 53, p. 63- 68, 2017. Disponível em:https://www.portaldoenvelhecimento.com/revistanova/index.php/revistaportal/article/viewFile/681/750.

MARQUES, L. P.; SCHNEIDER, I. J. C.; D'ORSI, E. Quality of life and its association with work, the Internet, participation in groups and physical activity among the elderly from the EpiFloripa survey, Florianópolis, Santa Catarina State, Brazil. Caderno de Saúde Pública, v. 32, n. 12, p. 1-11, 2016. Disponível em: $<$ http://www.scielo.br/scielo.php?script=sci_arttext\&pid=S0102-

311X2016001205009>. doi: http://dx.doi.org/10.1590/0102-311x00143615. 
MEDEIROS, F. de L.; XAVIER, A. J.; SCHNEIDER, I. J. C.; RAMOS, L. R.; SIGULEM, D. et al. Inclusão digital e capacidade funcional de idosos residentes em Florianópolis, Santa Catarina, Brasil (EpiFloripa 2009-2010). Revista Brasileira de Epidemiologia, v. 15, n. 1, p. 106-122, 2012. Disponível em: $<$ http://www.scielo.br/scielo.php?script=sci_arttext\&pid=S1415-

790X2012000100010 >. doi: http://dx.doi.org/10.1590/S1415-790X2012000100010.

MESSIAS, A. R. O idoso no Facebook: sociabilidade e encontro geracional. In: PORTO, C.; SANTOS, E. organizadores. Facebook e educação: publicar, curtir, compartilhar [online]. Campina Grande: EDUEPB, 2014, p. 237-251.

MIRANDA, L. M. de; FARIAS, S. F. As contribuições da internet para o idoso: uma revisão de literature. Interface - Comunicação Saúde Educação, v. 13, n. 29, p.383- 394, 2009. Disponível em: <http://www.scielo.br/pdf/icse/v13n29/v13n29a11.pdf>.

doi: http://dx.doi.org/10.1590/S1414-32832009000200011.

ORDONEZ, T. N.; LIMA-SILVA, T. B.; YASSUDA, M. S.; CACHIONI, M. Idosos on line: exemplo de metodologia de inclusão digital. Revista Temática Kairós Gerontologia, v. 15, n. 7, p. 215-234, 2012. Disponível em: https://revistas.pucsp.br/kairos/article/view/15250/11376.

OMS - Organização Mundial de Saúde. Relatório mundial de envelhecimento e saúde. Genebra: OMS, 2015. 28p. Acesso em: 10 janeiro. 2019. Disponível em < http://apps.who.int/iris/bitstream/handle/10665/186468/WHO_FWC_ALC_15.01_por. pdf;jsessionid=676DF0715DFD79344DA0917BE0C609AA? sequence $=6>$.

PEREIRA, C.; NEVES, R. Os idosos e as Novas tecnologias: competências e qualidade de vida. Revista Kairós Gerontologia, v.14, n. 1, p. 5- 26, 2011. Disponível em: https://revistas.pucsp.br/index.php/kairos/article/viewFile/7099/5139.

PESSOA, S. C.; VIEIRA, D. de A.; CAVALCANTI, F. I. D. A Internet: um espaço de sociabilidades para a terceira idade. Revista Gaúcha de Enfermagem, v. 29, n. 4, p. 654-658, 2008. Disponível em: https://seer.ufrgs.br/RevistaGauchadeEnfermagem/article/view/7639/4694.

PETERSEN, D. A. W.; KALEMPA, V. C.; PYKOSZ, L. C. Envelhecimento e Inclusão digital. Revista Extensio, v. 10, n. 15, p. 120-128, 2013. Disponível em: $<$ https://periodicos.ufsc.br/index.php/extensio/article/view/1807-

0221.2013v10n15p120>. doi: https://doi.org/10.5007/1807-0221.2013v10n15p120.

PEW RESEARCH CENTER. Social Media Fact Sheet, Washington, 5 fev. 2018. Disponível em: <http://www.pewinternet.org/fact-sheet/social-media/>.

PICON, F.; KARAM, R.; BREDA, V.; RESTANO, A.; SILVEIRA, A. et al. Precisamos falar sobre tecnologia: caracterizando clinicamente os subtipos de dependência de tecnologia. Revista Brasileira de Psicoterapia, v. 17, n. 2, p. 44-60, 2015. Disponível em: http://rbp.celg.org.br/detalhe_artigo.asp?id=177. 
SALES, M. B. de; AMARAL, M. A.; JUNIOR, I. G. S.; SALES, A. B. de. Tecnologias de informação e comunicação via web: preferências de uso de um grupo de usuários idosos. Revista Kairós Gerontologia, v. 17, n. 3, p. 59- 77, 2014. Disponível em: $<$ https://revistas.pucsp.br/kairos/article/view/21507/15756>.

SANTOS, L. G. N. de O.; ISHITANI, L.; NOBRE, C. N. Uso de jogos casuais em celulares por idosos: um estudo de usabilidade. Revista de Informática Aplicada, São Caetano do Sul, v. 9, n. 1, p. 24-44, 2013. Disponível em: $<$ http://ria.net.br/index.php/ria/article/view/88>.

doi: http://dx.doi.org/10.13037/ras.vol9n1.88.

SANTOS, T. S.; BRITO, T.A.; YOKOYAMA FILHO, F.S.; GUIMARÃES, L.A.; SOUTO, C.S.; SOUZA, S.J.N. et al. Desenvolvimento de um aplicativo para dispositivos móveis para identificar o fenótipo de fragilidade entre idosos. Revista Brasileira de Geriatria e Gerontologia, Rio de Janeiro, v. 20, n. 1, p. 70-76, jan./fev. $2017 . \quad$ Disponível em: <http://www.scielo.br/scielo.php?script=sci_arttext\&pid=S180998232017000100067\&lng=en\&tlng=en>. doi: http://dx.doi.org/10.1590/1981-22562017020.160025.

SILVA, D. A. S.; PEREIRA, M. M. O.; FERREIRA, M. C. Terceira idade e tecnologia: um estudo sobre a utilização da internet e do comércio eletrônico. Revista Brasileira de Gestão e Engenharia, v. 12, p. 61- 87, 2015. Disponível em: http://periodicos.cesg.edu.br/index.php/gestaoeengenharia/article/view/230/334.

SILVEIRA, B. O.; PARRIÃO, G. B. L.; FRAGELLI, R. R. Melhor idade conectada: um panorama da interação entre idosos e tecnologias móveis. Revista Tecnologias em Projeção, Brasília, v. 8, n. 2, p. 42-53, 2017. Disponível em: http://revista.faculdadeprojecao.edu.br/index.php/Projecao4/article/view/1004/836.

SKURA, I.; VELHO, A. P. M.; FRANCISCO, C. C. B. Mídias sociais digitais e a terceira idade: em busca de uma ferramenta para a promoção da saúde. Revista Kairós Gerontologia, v. 16, n. 4, p. 237-249, 2013. Disponível em: https://revistas.pucsp.br/kairos/article/view/19679/14568.

SOUZA, J. J. de; SALES, M. B. de. Tecnologias da informação e comunicação, smartphones e usuários idosos: uma revisão integrativa à luz das teorias sociológicas do envelhecimento. Revista Kairós Gerontologia, v. 19, n. 4, p. 131154, 2016. Disponível em: https://revistas.pucsp.br/kairos/article/view/31957/22141.

TAVARES, M. M. K.; SOUZA, S.T. C. de. Os idosos e as barreiras de acesso às novas tecnologias da informação e comunicação. Revista Novas Tecnologias na Educação, v. 10, n. 1, p. 1-7, 2012. Disponível em: https://seer.ufrgs.br/renote/article/viewFile/30915/19244.

WASSERMAN, C.; GRANDE, T. P. F.; MACHADO, L. R.; BEHAR, P. A. Redes Sociais: Um novo mundo para o idoso. Revista Novas Tecnologias na Educação, v. $1, \quad$ n. $10, \quad$ p. 1-10, 2012. Disponível em: $<$ https://seer.ufrgs.br/renote/article/view/30863/19222>. doi: https://doi.org/10.22456/1679-1916.30863 